\title{
Massive spin 3/2 electrodynamics
}

\author{
S. Deser* and A. Waldron ${ }^{\dagger}$ \\ Physics Department, Brandeis University, Waltham, Massachusetts 02454 \\ V. Pascalutsa \\ Department of Physics, Flinders University, Bedford Park, SA 5042, Australia
}

(Received 3 March 2000; published 26 October 2000)

\begin{abstract}
We study the general non-minimally coupled charged massive spin $3 / 2$ model both for its low energy phenomenological properties and for its unitarity, causality and degrees of freedom behavior. When the model is viewed as an effective theory, its parameters (after ensuring the correct excitation count) are related to physical characteristics, such as the magnetic moment $g$ factor, by means of low energy theorems. We also provide the corresponding higher spin generalization. Separately, we consider both low and high energy unitarity, as well as the causality of our models. None (including truncated $N=2$ supergravity) is free of the minimal model's acausality.
\end{abstract}

PACS number(s): 11.10.Ef, 04.65.+e, 13.40.Em, 14.80.-j

\section{INTRODUCTION}

Gauge interactions of massive (let alone massless) relativistic higher spin fields constitute an ancient and difficult subject. Whatever the formal problems these models encounter, effective higher spin theories must be constructible since approximately localized higher spin particles exist. Such models should achieve low energy consistency, and share some of the physical properties described by their lower spin hadronic physics counterparts.

In our present study of charged massive higher spins we seek only effective (rather than renormalizable) actions, which can in general possess dimensionful, non-minimal, couplings beyond the minimal prescription, unique in first order systems, $\partial_{\mu} \rightarrow \partial_{\mu}+i e A_{\mu}$. In particular, the associated coupling constants will determine $2 s+1$ intrinsic multipole moments of a spin $s$ particle (charge, magnetic dipole, quadrupole and octupole for $s=3 / 2$ ).

The more formal properties, such as unitarity and causality, of higher spin models will in general also depend upon details of the non-minimal couplings. Some of the important affected issues include: (i) A gyromagnetic ratio $g=2$ is required by the optical and low energy theorems, at least for pure electromagnetic interactions [1], on the other hand, minimal coupling implies $g=2 / 3$ [2]. (ii) Tree unitarity [3] requires the non-minimal couplings of (truncated) $N=2$ supergravity [4]. (iii) Quantization of the minimal theory is problematic since the fundamental canonical commutator becomes indefinite beyond a critical value of the magnetic field [5], or equivalently the model exhibits acausal propagation [6]. However, if the minimal electromagnetic interactions are extended to include gravity as obtained by dropping only the cosmological constant term of $N=2$ anti-de Sitter supergravity [7], causality is restored [8]. Unfortunately, this formally consistent model is unsuitable for phenomenological

\footnotetext{
*Email address: deser@brandeis.edu

†Email address: wally@brandeis.edu

*Email address: phvvp@ flinders.edu.au
}

applications, since it tunes spin $3 / 2$ and Planck masses.

In this paper, we study the low energy behavior, unitarity and causal consistency of general flat space non-minimal, non-derivative, couplings, concentrating on the massive charged spin $3 / 2$ system, the simplest theory subject to the array of higher spin subtleties. Of the five independent nonminimal terms linear in the field strength, two are eliminated by a simple degrees of freedom (DOF) consistency requirement. One of the three remaining couplings does not contribute to photon emission and Compton scattering but is included in our causality analysis. We employ low energy theorems (LETs) to identify the leading low energy nonminimal coupling as a magnetic moment interaction and compute the gyromagnetic ratio $g$ in terms of the parameters in the action. The generalization of this result to higher spins is given in Appendix A.

The gyromagnetic ratio $g$ in our models is arbitrary and thus they are perfectly suited for phenomenological applications where neither the pure electromagnetic $g=2$ unitarity requirement nor tree unitarity need apply. Loss of the latter merely signals the scale at which the effective description ceases to be valid.

Study of causality yields a negative result; like the minimal model, ours all permit acausal propagation for critical electromagnetic fields. This result applies to arbitrary (DOFpreserving) non-minimal couplings and agrees with an old result [9] valid for couplings linear in the field strength. (In [8] causality is preserved by taking gravity and electromagnetism not merely external, but dynamical; curved space will be included elsewhere [10].) Nevertheless, we will argue that for perturbative processes, formulated in terms of free asymptotic fields, neither high energy unitarity nor causality problems spoil the validity of the models as a phenomenological tool.

In Sec. II we present the non-minimal models under consideration and obtain the constraints required by a correct DOF count. Section III contains the verification of the LETs for the soft photon vertex and Compton scattering, along with our gyromagnetic ratio computation. A study of causality is presented in Sec. IV. In Sec. V we summarize and 
discuss our work. The generalization of our soft photon vertex results and identification of the magnetic moment for higher spins is given in Appendix A and the extension of our causality analysis to the most general non-minimal couplings is given in Appendix B.

\section{THE MODELS}

We begin with the Lagrangian for the complex vectorspinor Rarita-Schwinger field $\psi_{\mu}$,

$$
\begin{aligned}
\mathcal{L}= & -\bar{\psi}^{\mu} \gamma_{\mu \nu \rho} \mathcal{D}^{\nu} \psi^{\rho}-\frac{i e}{m} \bar{\psi}_{\mu} \mathcal{F}^{\mu \nu} \psi_{\nu} ; \\
\bar{\psi}_{\mu} \mathcal{F}^{\mu \nu} \psi_{\nu} \equiv & l_{1} \bar{\psi}_{\mu} F^{\mu \nu} \psi_{\nu}+l_{2} \bar{\psi}_{\mu} \hat{F} \psi^{\mu} \\
& +l_{3} F^{\mu \nu}\left[\bar{\psi}_{\mu} \gamma_{\nu} \gamma \cdot \psi+\bar{\psi} \cdot \gamma \gamma_{\mu} \psi_{\nu}\right] \\
& +l_{4} \bar{\psi} \cdot \gamma \hat{F} \gamma \cdot \psi \\
& +i l_{5} F^{\mu \nu}\left[\bar{\psi}_{\mu} \gamma_{\nu} \gamma \cdot \psi-\bar{\psi} \cdot \gamma \gamma_{\mu} \psi_{\nu}\right]
\end{aligned}
$$

Our conventions are: Metric $\eta_{\mu \nu}=\operatorname{diag}(-,+,+,+)$, $(\mu, \nu, \ldots=0, \ldots, 3, i, j, \ldots=1,2,3) ;$ Dirac matrices: $\left\{\gamma^{\mu}, \gamma^{\nu}\right\}=2 \eta^{\mu \nu}, \quad \gamma^{\mu \dagger}=\gamma^{0} \gamma^{\mu} \gamma^{0} ; \quad \gamma^{\mu_{1}} \ldots \mu_{n} \equiv \gamma^{\left[\mu_{n}\right.} \ldots \gamma^{\left.\mu_{n}\right]}$ [we always (anti)symmetrize with unit weight]; $\gamma^{5}=$ $-i \gamma^{0123}, \gamma^{\mu \nu \rho \sigma}=i \gamma^{5} \epsilon^{\mu \nu \rho \sigma}$. Contraction of all indices of a tensor with Dirac matrices is denoted by a hat, e.g., $\hat{F}$ $=F_{\mu \nu} \gamma^{\mu} \gamma^{\nu}$. The operator in the minimal term of Eq. (1),

$$
\mathcal{D}_{\mu} \equiv D_{\mu}+\frac{1}{2} m \gamma_{\mu}, \quad D_{\mu} \psi_{\nu}=\partial_{\mu} \psi_{\nu}+i e A_{\mu} \psi_{\nu},
$$

also incorporates the usual mass term $m \bar{\psi}_{\mu} \gamma^{\mu \nu} \psi_{\nu}$; it satisfies

$$
\left[\mathcal{D}_{\mu}, \mathcal{D}_{\nu}\right]=i e F_{\mu \nu}+\frac{1}{2} m^{2} \gamma_{\mu \nu}, \quad\left[\mathcal{D}_{\mu}, \gamma_{\nu}\right]=m \gamma_{\mu \nu}
$$

Note that $i l_{5} F^{\mu \nu}\left[\bar{\psi}_{\mu} \gamma_{\nu} \gamma \cdot \psi-\bar{\psi} \cdot \gamma \gamma_{\mu} \psi_{\nu}\right]$, being diagonal in a Majorana basis, is non-vanishing even for an uncharged real field; on-shell (where $\gamma \cdot \psi=0$ ) it does not contribute at lowest perturbative orders and we therefore drop it until the general causality analysis in Sec. IV.

The set (2) represents the most general non-derivative, Hermitian, parity-even couplings linear in the field strength $F_{\mu \nu}$. That they must constitute a five parameter family can also be seen upon expanding the most general possible $\bar{\psi}_{\mu} \Gamma^{\mu \nu \rho \sigma} \psi_{\nu} F_{\rho \sigma}$ in a Fierz basis: There is a single coupling to $\gamma^{\mu \nu \rho \sigma}$, three possibilities for $\gamma^{\mu \nu}$ and a single scalar 1 coupling. Thus the $\gamma^{5} \widetilde{F}^{\mu \nu}$ of supergravity $\left[\widetilde{F}^{\mu \nu}\right.$ $\left.\equiv(1 / 2) \epsilon^{\mu \nu \rho \sigma} F_{\rho \sigma}\right]$ may be cast in the above basis as

$$
\begin{aligned}
\bar{\psi}_{\mu} i \gamma^{5} \widetilde{F}^{\mu \nu} \psi_{\nu}= & \bar{\psi}_{\mu} F^{\mu \nu} \psi_{\nu}-\frac{1}{2} \bar{\psi}_{\mu} \hat{F} \psi^{\mu} \\
& -F^{\mu \nu}\left[\bar{\psi}_{\mu} \gamma_{\nu} \gamma \cdot \psi+\bar{\psi} \cdot \gamma \gamma_{\mu} \psi_{\nu}\right] \\
& +\frac{1}{2} \bar{\psi} \cdot \gamma \hat{F} \gamma \cdot \psi
\end{aligned}
$$

Two of the five parameters in Eqs. (1),(2) may be eliminated by requiring that the model describe the correct DOF or, equivalently, maintains the constraint count of the free theory: The zeroth component of the equation of motion $R_{0}$ $=\delta \mathcal{L} / \delta \bar{\psi}^{0}$ involves no time derivatives and is therefore a constraint eliminating four of the sixteen (complex) components of $\psi_{\mu}$. Another constraint eliminating four more components is still required before one can conclude that on-shell half of the remaining components yield $2 s+1=4$ physical DOF. When $\psi_{0}$ appears linearly in the action, as for the minimal theory, it is a Lagrange multiplier imposing the constraint $R_{0}=0$. Requiring its preservation under time evolution, $\dot{R}_{0}=0$, yields the necessary additional constraint. If instead $\psi_{0}$ appears quadratically (i.e., as $\psi_{0}^{\dagger} M \psi_{0}$ for some matrix $M$ ) the $R_{0}=0$ equation now determines $\psi_{0}$, and requiring $\dot{R}_{0}=0$ yields an equation of motion for $\dot{\psi}_{0}$. This choice describes too few constraints-too many (propagating) DOF, as compared to the free field.

One non-minimal model respecting the DOF count is the truncation of $N=2$ supergravity with the cosmological, curvature and four-Fermi terms omitted. ${ }^{1}$ It corresponds by Eq. (5) to the choice of parameters $l_{1}=-2, l_{2}=1 / 2, l_{3}=1$, $l_{4}=-1 / 2\left(\right.$ and $\left.l_{5}=0\right)$ reproducing $\mathcal{F}_{\mu \nu}=-\left(F_{\mu \nu}+i \gamma^{5} \widetilde{F}_{\mu \nu}\right)$. (If however, as in [4], the further truncation excluding the gamma-trace components of $\psi_{\mu}$ in the non-minimal sector is made, the DOF count is violated. This fact would seem to make moot the causality claim there.)

Henceforth, we retain only models linear in $\psi_{0}$, which, as is easily seen, is equivalent to demanding $\mathcal{F}_{\mu \nu}=-\mathcal{F}_{\nu \mu}$. (Since any timelike vector $\xi_{\mu}$ defines a time direction, we may rephrase the requirement that $\psi_{0}$ appear only linearly in Lorentz covariant language as $\xi \cdot \mathcal{F} \cdot \xi=0$ for all $\xi_{\mu}$ timelike.) The corresponding relations amongst parameters are

$$
l_{2}+l_{4}=0, \quad l_{3}+2 l_{4}=0,
$$

and the non-minimal interactions reduce to the two combinations (dropping $l_{5}$ )

$$
\bar{\psi}_{\mu} \mathcal{F}^{\mu \nu} \psi_{\nu}=\left[l_{1}+2 l_{2}\right] \bar{\psi}_{\mu} F^{\mu \nu} \psi_{\nu}-2 l_{2} \bar{\psi}_{\mu} i \gamma^{5} \widetilde{F}^{\mu \nu} \psi_{\nu}
$$

\footnotetext{
${ }^{1}$ This truncation should not be confused with the supersymmetrypreserving anti-de Sitter-Poincare contraction, mapping the model of [7] to the original $N=2$ model [11]. The latter has a flat gravitational background and only non-minimal, uncharged, Maxwell couplings.
} 
We now consider the properties of these physical spin $3 / 2$ theories. The model with $l_{1}=0=l_{2}=l_{3}=l_{4}=l_{5}$ will be referred to as the minimal one while "supergravity-inspired model" refers to the just-described truncated $N=2$ supergravity.

\section{LOW ENERGY THEOREMS}

LETs characterize soft photon scattering amplitudes in terms of the mass, charge and magnetic moment of the target $[12,13,1]$, independent of its internal structure, relying only on gauge and Lorentz invariance plus low photon frequency. In this sense, LETs are purely kinematical and, irrespective of any causal pathologies, perturbative scattering amplitudes formulated in terms of free asymptotic fields are guaranteed to satisfy them. Therefore LETs provide a simple way to map the QFT parameters to the physical ones.

We first study the vertex for the emission of a single low frequency photon, then Compton scattering with small incoming (and outgoing) photon frequencies. In each case the relevant LETs are usually stated for stationary targets and Lorentz invariance is not manifest. So we first enunciate the dictionary to our relativistic Feynman tree amplitudes. A soft photon is invariantly defined by the requirement that

$$
\frac{\omega^{a}}{m} \equiv-\frac{p \cdot k^{a}}{m^{2}} \ll 1,
$$

for photon a's four-momentum $k_{\mu}^{a}$ and target $p_{\mu}$. In the laboratory frame $p_{\mu}=(m, 0,0,0), \omega^{a}$ reduces to the usual photon frequency.

For each photon polarization $\varepsilon_{\mu}^{a}$ we employ Feynman gauge $k^{a} \cdot \varepsilon^{a}=0$ along with the residual gauge fixing conditions $p \cdot \varepsilon^{a}=0$ so that in the laboratory frame one has $\varepsilon_{\mu}^{a}$ $=\left(0, \vec{\epsilon}^{a}\right)$ and $\vec{k}^{a} \cdot \vec{\epsilon}^{a}=0$. We also utilize a covariant notation for the target polarizations $u_{\mu}=u_{\mu}(p)$ and $\bar{u}_{\mu}^{\prime}=\bar{u}_{\mu}\left(p^{\prime}\right)$ where $p_{\mu}^{\prime}$ is the outgoing momentum of the target particle and the usual asymptotic on-shell conditions hold

$$
\begin{gathered}
p \cdot u=\gamma \cdot u=0=(i p+m) u_{\mu}, \\
\bar{u}^{\prime} \cdot p^{\prime}=\bar{u}^{\prime} \cdot \gamma=0=\bar{u}_{\mu}^{\prime}\left(i p^{\prime}+m\right) .
\end{gathered}
$$

An explicit representation for the spin $3 / 2$ polarizations in terms of the usual massive spin 1 and spin $1 / 2$ polarizations $\varepsilon_{\mu}^{l}(l=-1,0,1)$ and $u^{s}(s=-1 / 2,1 / 2)$, respectively, is given by $u_{\mu}^{ \pm 3 / 2}=\varepsilon_{\mu}^{ \pm 1} u^{ \pm 1 / 2}$ and $u_{\mu}^{ \pm 1 / 2}=\left(\varepsilon_{\mu}^{ \pm 1} u^{\mp 1 / 2}\right.$ $\left.+\sqrt{ } 2 \varepsilon_{\mu}^{0} u^{ \pm 1 / 2}\right) / \sqrt{ } 3$ [14]. Obviously, inserting $u_{\rho}=u_{\rho}^{\lambda}$ and $\bar{u}_{\rho}^{\prime}$ $=\bar{u}_{\rho}^{\lambda^{\prime}}$ with $\lambda, \lambda^{\prime}=-3 / 2, \ldots, 3 / 2$ in Eq. (12) below, $\mathcal{M}_{\lambda^{\prime} \lambda}^{\mu \nu}$ (regarded as a matrix in the labels $\lambda$ and $\lambda^{\prime}$ ) is a spin $3 / 2$ irreducible representation of the Lorentz algebra.

The total spin matrix $\vec{S}$ is the dual of the spatial Lorentz generators, $S^{i}=(i / 2) \epsilon^{i j k} M_{j k}$, and the Lorentz generators act on the relativistic vector-spinor on-shell representation of the spin $3 / 2$ polarizations $u_{\rho}$ according to

$$
M^{\mu \nu, \rho}{ }_{\sigma}=\frac{1}{2} \gamma^{\mu \nu} \delta_{\sigma}^{\rho}+2 \delta^{[\mu|\rho|} \delta^{\nu]}{ }_{\sigma}
$$

so that

$$
\delta_{\text {Lorentz }} u_{\rho}=\frac{1}{2} \lambda_{\mu \nu} M^{\mu \nu}, \rho^{\sigma} u_{\sigma}=\lambda_{\rho}{ }^{\sigma} u_{\sigma}+\frac{1}{4} \hat{\lambda} u_{\rho} .
$$

It is useful to define

$$
\begin{aligned}
\mathcal{M}^{\mu \nu} & \equiv \bar{u}_{\rho}^{\prime} M_{\sigma}^{\mu \nu, \rho} u^{\sigma}=\frac{1}{2} \bar{u}_{\rho}{ }_{\rho} \gamma^{\mu \nu} u^{\rho}+2 \bar{u}^{\prime}{ }^{[\mu} u^{\nu]}, \\
\mathcal{S}^{i} & \equiv \frac{i}{2} \epsilon^{i j k} \bar{u}_{\rho}^{\prime} M_{j k}{ }^{\rho}{ }_{\sigma} u^{\sigma} .
\end{aligned}
$$

The LET for the photon vertex states that the amplitude for emission of a soft photon by a stationary mass $m$, spin $s$ target is

$$
T_{f i}=-\frac{i \mu}{s}(\vec{\epsilon} \times \vec{k}) \cdot \overrightarrow{\mathcal{S}}+\mathcal{O}\left(\omega^{2}\right)
$$

transparent derivations of Eqs. (13) and (15) may be found in [1]. The magnetic moment $\mu$ appearing in Eq. (13) is related to the charge to mass ratio of a spin $s$ particle by the gyromagnetic ratio $g$, defined by

$$
\mu \equiv \frac{e g s}{2 m} .
$$

The standard LET for Compton scattering reads

$$
\begin{aligned}
T_{f i}= & -\frac{e^{2}}{m} \vec{\epsilon}^{\prime} \cdot \vec{\epsilon} \vec{u}^{\prime} \cdot u+\frac{i e \omega}{m}\left(\frac{2 \mu}{s}-\frac{e}{m}\right)\left(\vec{\epsilon}^{\prime} \times \overrightarrow{\boldsymbol{\epsilon}}\right) \cdot \overrightarrow{\mathcal{S}} \\
& -\frac{i e \mu}{\omega s}\left(\vec{\epsilon} \cdot \vec{k}^{\prime}\left(\vec{\epsilon}^{\prime} \times \vec{k}^{\prime}\right)-\overrightarrow{\boldsymbol{\epsilon}} \cdot \vec{k}(\overrightarrow{\boldsymbol{\epsilon}} \times \vec{k})\right) \cdot \overrightarrow{\mathcal{S}} \\
& -\frac{i \mu^{2}}{\omega s^{2}}\left(\left(\vec{\epsilon}^{\prime} \times \vec{k}^{\prime}\right) \times(\overrightarrow{\boldsymbol{\epsilon}} \times \vec{k})\right) \cdot \overrightarrow{\mathcal{S}}+\mathcal{O}\left(\omega^{2}\right) .
\end{aligned}
$$

Our task now is to derive the amplitudes (13) and (15) in a Lagrangian framework and thereby relate the parameters of Eqs. (1),(7) to the physical ones which (apart from $e$ and $m$ ) means the single number $g$.

\section{A. The soft photon vertex}

The amplitude (13) may, using the on-shell conditions for the target and soft photon polarizations, be expressed in the manifestly Lorentz invariant form

$$
T_{f i}=\frac{i \mu}{2 s} F_{\mu \nu} \mathcal{M}^{\mu \nu}+\mathcal{O}\left(\omega^{2}\right), \quad F_{\mu \nu}=i\left(k_{\mu} \epsilon_{\nu}-k_{\nu} \epsilon_{\mu}\right) .
$$

On-shell the interaction Lagrangian, including minimal and non-minimal couplings, becomes 


$$
\begin{aligned}
\left.i \mathcal{L}^{\text {int }}\right|_{\text {on-shell }}= & i T_{f i}=e \bar{u}_{\rho} \gamma \cdot \epsilon u^{\rho} \\
& +\frac{e}{m}\left[l_{1} \bar{u}_{\mu} F^{\mu \nu} u_{\nu}+l_{2} \bar{u}_{\rho} \hat{F} u^{\rho}\right] .
\end{aligned}
$$

The asymptotic Rarita-Schwinger equation may be used to derive the obvious generalization

$$
\begin{aligned}
\bar{u}_{\rho}^{\prime}\left(p^{\prime}\right) \gamma^{\mu} u_{\sigma}(p)= & -\frac{i}{2 m}\left(p+p^{\prime}\right)^{\mu} \bar{u}_{\rho}^{\prime} u_{\sigma} \\
& +\frac{i}{2 m}\left(p^{\prime}-p\right)_{\nu} \bar{u}_{\rho}^{\prime} \gamma^{\mu \nu} u_{\sigma}
\end{aligned}
$$

of the spin 1/2 Gordon identity. Thus the amplitude derived from the non-minimal Lagrangian reads

$$
T_{f i}=-\frac{i e}{2 m}\left(2 l_{1} \bar{u}_{\mu} F^{\mu \nu} u_{\nu}+\frac{1}{2}\left(4 l_{2}-1\right) \bar{u}_{\rho} \hat{F} u^{\rho}\right) \text {. }
$$

At this juncture, the amplitude seems quite different from that of a pure $s=1 / 2$ system for which there is a one parameter family of $(g-2)$ values read off from the $\hat{F}$ term with, of course, no counterpart to the $l_{1}$ term. If the latter is to augment the $\hat{F}$ term to a coupling involving the full Lorentz generators as in Eq. (12), the relevant coefficient between the two terms must be $1 / 4$. There must be, therefore, an identity relating these two terms for the LET to hold; LETs are just a statement about parts of amplitudes determined completely by kinematics and should be reproduced for any choice of parameters. ${ }^{2}$ The on-shell identity ${ }^{3}$

$$
\bar{u}_{\rho}^{\prime}\left(p^{\prime}\right) \gamma_{\mu \nu} u^{\rho}(p)=2 \bar{u}_{[\mu}^{\prime}\left(p^{\prime}\right) u_{\nu]}(p)+\mathcal{O}(\omega)
$$

is easily verified in the frame $p_{\mu}=(m, 0,0,0)$. This low energy equality states that on-shell the spin $1 / 2$ and spin 1 parts

\footnotetext{
${ }^{2}$ In [7] the Thompson limit for Compton scattering was obtained in the truncated $N=2$ supergravity model, a calculation that seemed to hinge on delicate cancellations due to the particular form of the supergravity non-minimal couplings. However, since the Thompson limit is dictated by the lowest order LET, our generic computation always guarantees this result.

${ }^{3}$ It is already possible without using the identity (20), to directly satisfy the LET with the choice of parameters $l_{1}=-g / 2=4 l_{2}-1$ : The Lagrangian becomes $\quad \mathcal{L}=\mathcal{L}_{\text {min }}+(i e / m) \bar{\psi}_{\mu} F^{\mu \nu} \psi_{\nu}+[i e(g$ $-2) / 4 m] F_{\mu \nu} \bar{\psi}_{\rho} M^{\mu \nu, \rho}{ }_{\sigma} \psi^{\sigma}+[i e(g-2) / 4 m]\left(F^{\mu \nu}\left[\bar{\psi}_{\mu} \gamma_{\nu} \gamma \cdot \psi\right.\right.$ $\left.\left.+\bar{\psi} \cdot \gamma \gamma_{\mu} \psi_{\nu}\right]-\frac{1}{2} \bar{\psi} \cdot \gamma \hat{F} \gamma \cdot \psi\right)$. The LET is then satisfied by the following interesting mechanism: Together, the first two terms of $\mathcal{L}$ produce a $g=2$ coupling since the minimal Lagrangian gives the spin $1 / 2$ part of the Lorentz generators via the Gordon identity above and the non-minimal coupling to $F^{\mu \nu}$ yields the spin 1 contribution (exactly the same term required for a spin 1 vector boson to have $g=2$ ). The third term is a direct coupling to the total Lorentz generators and yields an anomalous magnetic moment coupling (the remaining ones are required to ensure the correct DOF in the $g \neq 2$ case)
}
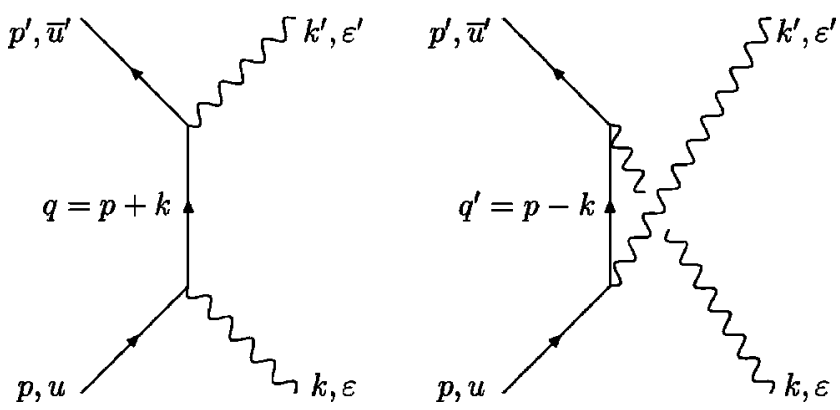

FIG. 1. Compton scattering kinematics.

of the Lorentz generators may be traded against one another. Hence the low energy result is precisely reproduced by any of the non-minimal Lagrangians, the gyromagnetic ratio being

$$
g=\frac{2}{3}-\frac{4}{3}\left(l_{1}+2 l_{2}\right)
$$

a sum of minimal and non-minimal contributions. For minimal coupling the well known result $g=1 / s=2 / 3$ emerges [2]. Observe that the supergravity-inspired model yields $g=2$ [4]. Finally, we note, in passing, that in the basis (7) only the $F_{\mu \nu}$ coupling contributes to the gyromagnetic ratio (21), independent of the $i \gamma^{5} \widetilde{F}_{\mu \nu}$ term. This is not surprising since $\gamma^{5}$ mixes the "large" and "small" components of the vectorspinor $u_{\mu}$ defined by projection with respect to $i \gamma^{0}$, and is of higher order in the soft photon expansion. This term will contribute to higher quadrupole and octupole moments. The extension of our work to such moments is an interesting but separate issue.

\section{B. Compton scattering}

A useful check on our vertex result (21) is to compute the amplitude of Fig. 1 for Compton scattering. In particular, since the gyromagnetic ratio must be precisely $g=2$ for the optical theorem to hold [1], the latter will produce an additional relation between the parameters $l_{1}$ and $l_{2}$.

The mass shell condition $p^{\prime 2}=-m^{2}$ implies that the difference $\omega^{\prime}-\omega=k \cdot k^{\prime} / m$ is second order in this expansion. Therefore we eliminate $\omega^{\prime}$ (using this relation) and $p^{\prime}$ (by momentum conservation); one can then evaluate the order of any expression simply by counting the number of fourvectors $k$ and $k^{\prime}$ in it. We now evaluate [15] the relevant $s$ and $u$ channel diagrams in this limit, using the vertices of Eq. (1) and the free propagator

$$
\begin{aligned}
S_{\mu \nu}^{F}(p)= & \frac{-i}{p^{2}+m^{2}}\left[\left(\eta_{\mu \nu}+\frac{p_{\mu} p_{\nu}}{m^{2}}\right)(i p-m)+\frac{1}{3}\left(\frac{i p_{\mu}}{m}-\gamma_{\mu}\right)\right. \\
& \left.\times(i p+m)\left(\frac{i p_{\nu}}{m}-\gamma_{\nu}\right)\right] .
\end{aligned}
$$

To extract the leading and next-to-leading terms in the low energy expansion of the amplitude, we need the Gordon identity (18) of the previous section along with the following generalization: 


$$
\begin{aligned}
\bar{u}_{\lambda}^{\prime}\left(p^{\prime}\right) \gamma^{\mu \nu \rho} u_{\kappa}(p)= & -\frac{3 i}{2 m}\left(p+p^{\prime}\right)^{[\mu} \bar{u}_{\lambda}^{\prime} \gamma^{\nu \rho]} u_{\kappa} \\
& +\frac{i}{2 m} \bar{u}_{\lambda}^{\prime} \gamma^{\mu \nu \rho \sigma} u_{\kappa}\left(p^{\prime}-p\right)_{\sigma} .
\end{aligned}
$$

Note that in the laboratory frame, the "Dirac" equation (9) reads $\gamma^{0} u=i m u$ and using the above Gordon identities, expressions such as $\bar{u}_{\rho}^{\prime} \gamma^{\mu \nu} \varepsilon_{\mu} k_{\nu} u_{\sigma}$ are effectively equal to $\bar{u}_{\rho}^{\prime} \gamma^{i j} \epsilon_{i} k_{j} u_{\sigma}$ at leading order in the low energy expansion. As a simple gauge invariance check, before imposing the residual gauge choice $p \cdot \varepsilon=0=p \cdot \varepsilon^{\prime}$ and taking the low energy limit, we verified that our amplitude satisfies transversality in each photon line separately.

Once again, applying only the Gordon identities (18) and (23), the amplitude satisfies the LET only for the choice of parameters of the Lagrangian of footnote 3. However, once one applies further the on-shell identity (20) along with the additional identity [equivalent to Eq. (20) upon contracting indices $\mu$ and $\sigma$ ]

$$
\bar{u}_{[\mu}^{\prime} \gamma_{\nu][\rho} u_{\sigma]}=\bar{u}_{[\rho}^{\prime} \gamma_{\sigma][\mu} u_{\nu]}+\mathcal{O}(\omega)
$$

one finds for the amplitude (in an obvious matrix notation for vector indices)

$$
\begin{aligned}
T_{f i}= & -\frac{e^{2}}{m} \bar{u}^{\prime} \cdot u \varepsilon^{\prime} \cdot \varepsilon+\frac{e^{2}}{4 m^{3}}(g-2)^{2} p \cdot k\left(\varepsilon^{\prime} \cdot \mathcal{M} \cdot \varepsilon\right) \\
& -\frac{i e^{2} g}{4 m p \cdot k}\left[\operatorname{tr}\left(F^{\prime} \cdot \mathcal{M}\right) \varepsilon \cdot k^{\prime}-\operatorname{tr}(F \cdot \mathcal{M}) \varepsilon^{\prime} \cdot k\right] \\
& +\frac{e^{2} g^{2}}{4 m p \cdot k} \operatorname{tr}\left(F^{\prime} \cdot \mathcal{M} \cdot F\right)+\mathcal{O}\left(\omega^{2}\right)
\end{aligned}
$$

where, as in the previous section $g$ stands for the combination (21) of parameters $g=2 / 3-4 / 3\left(l_{1}+2 l_{2}\right)$. It is not difficult to verify that in the laboratory frame, the amplitude (25) precisely reproduces the LET (15). Although this result is guaranteed by group theoretical arguments, our simple derivation is completely within the context of conventional Lorentz invariant quantum field theory. As such, it is a simple example of the model applied as an effective theory.

\section{Unitarity and $g=2$}

We close this section with some comments on unitarity. As mentioned, low energy unitarity imposes the value $g=2$ for any spin [1]. The idea is that, (for a particle interacting electromagnetically only) the optical theorem constrains the low energy forward $\left(k=k^{\prime}\right)$ limit of the scattering amplitude (15),

$$
\begin{aligned}
T_{f i}= & -\frac{e^{2}}{m} \vec{\epsilon}^{\prime} \cdot \overrightarrow{\boldsymbol{\epsilon}} \bar{u}^{\prime} \cdot u-\frac{i e^{2} \omega}{4 m^{2}}(g-2)^{2} \\
& \times\left(\overrightarrow{\boldsymbol{\epsilon}}^{\prime} \times \overrightarrow{\boldsymbol{\epsilon}}\right) \cdot \overrightarrow{\mathcal{S}}+\mathcal{O}\left(\omega^{2}\right),
\end{aligned}
$$

to have no contribution linear in $\omega$, thus requiring $g=2$. Clearly, if one wishes to apply this criterion to our model, one can simply take the choice of parameters $l_{1}+2 l_{2}=$ -1 . Of course, in reality, one may be interested in an effective description of a composite particle participating in the strong interactions with $g \neq 2$. Here the models with general values of $g$ are suitable.

The quite distinct criterion of tree unitarity $[3,16,4]$ concerns the high energy behavior of the theory. Partial wave amplitudes are subject to (constant) unitarity bounds which may, in principle, be violated by tree level amplitudes. For effective theories these bounds determine the energy scale at which the effective description fails and new physics enters [e.g., inapplicability of the Fermi weak interaction theory beyond $\left.m_{W}\right]$. The failure of tree unitarity in the minimal model was first observed in [16]. (Their inference of a connection between this and acausality seems unwarranted, however, given that the $g=2$ tree unitary model also fails to propagate causally.) Let us review the tree level unitarity argument $[16,4]$ in more detail. Massive higher spin propagators, e.g. (22), contain inverse powers of the mass. In tree level Green functions, for general kinematical configurations where all momenta are large, these terms lead to contributions growing with positive powers of the energy $E$. In addition to being dangerous for renormalizability when higher order loops are constructed from trees, they eventually violate partial wave unitarity bounds. There is, however, a quite general mechanism related to gauge invariance to remove this undesirable high energy behavior. Namely, if one investigates the worst powers of inverse mass in the propagator (22)

$$
\frac{2 p_{\mu} p p_{\nu}}{3 m^{2}\left(p^{2}+m^{2}\right)},
$$

one see that the operators $p_{\mu}$ and $p_{\nu}$ generate a linearized gauge transformation [in our case a linearized local supersymmetry transformation]

$$
\delta \psi_{\mu}=\partial_{\mu} \varepsilon
$$

at the vertices to which the propagator is attached. Hence requiring the on-shell vertices to satisfy the appropriate supersymmetric Ward identity will lead to cancellation of $1 / \mathrm{m}^{2}$ contributions. Obviously one can apply this procedure to further constrain the non-minimal couplings. The supergravityinspired model uniquely satisfies this criterion via the usual supersymmetry Ward identity. From a fundamental viewpoint this model may be favored, but amongst phenomenological effective theories, the most compelling choice of nonminimal coupling is dictated by the data [for example a measurement of the gyromagnetic ratio]. Any breakdown of tree unitarity simply indicates the scale at which the effective description is no longer valid. 


\section{CAUSALITY AND QUANTIZATION}

As we shall discuss, a study of causality ${ }^{4}$ amounts to investigating whether the constraints, required to ensure the correct physical DOF, are consistent. In particular, one may find that, for some critical value of the external field $F_{\mu \nu}$, the secondary Lagrangian constraint ${ }^{5}$ may no longer be inverted to solve for the Lagrange multiplier variable $\psi_{0}$. From a canonical viewpoint, this implies that the Dirac bracket governing dynamics on the constraint surface is ill-defined at this point [5] and yields a pathology that, of course, extends to the corresponding quantum mechanical canonical commutators. In terms of the field equations, this pathology implies that the model permits superluminal propagation [6]. A brief review of the precise relation between causality breakdown and consistency of constraints is given at the end of this section.

The key point is to find, and study the consistency of, eight (complex) constraints amongst the sixteen field components $\psi_{\mu}$; the equations of motion then reduce these to four physical DOF.

The field equation derived from Eq. (1) is

$$
R_{\mu} \equiv \delta S / \delta \bar{\psi}^{\mu}=\gamma_{\mu \nu \rho} \mathcal{D}^{\nu} \psi^{\rho}+\frac{i e}{m} \mathcal{F}_{\mu \nu} \psi^{\nu}=0
$$

Since $R_{0}$ does not involve time derivatives of any fields,

$$
\Theta_{1} \equiv \gamma^{0} R_{0}=\gamma_{i j} \mathcal{D}^{i} \psi^{j}+\frac{i e}{m} \gamma_{0} \mathcal{F}^{0 i} \psi_{i}=0
$$

is a primary constraint. As explained in Sec. II, a correct DOF count requires $\mathcal{F}_{00}=0$ in order that Eq. (30) does not determine the Lagrange multiplier $\psi_{0}$.

Before taking the divergence of $R_{\mu}$ to determine the secondary constraint we employ the relation [equivalent to Eq. (30) on-shell]

$$
\gamma \cdot R=2(\not D-3 m) \gamma \cdot \psi-2 \mathcal{D} \cdot \psi+\frac{i e}{m} \gamma \cdot \mathcal{F} \cdot \psi=0
$$

to rewrite the field equations $R_{\mu}$ as

$$
\begin{aligned}
R_{\mu}= & (\not \mathcal{D}-m) \psi_{\mu}-\left(\mathcal{D}_{\mu}-\gamma_{\mu}[\not \mathcal{D}-2 m]\right) \gamma \cdot \psi \\
& -\gamma_{\mu} \mathcal{D} \cdot \psi+\frac{i e}{m} \mathcal{F}_{\mu \nu} \psi^{\nu} \\
= & (\not D-m) \psi_{\mu}-\left(\mathcal{D}_{\mu}-m \gamma_{\mu}\right) \gamma \cdot \psi+\frac{i e}{2 m} \gamma_{\nu} \gamma_{\mu} \mathcal{F}^{\nu \rho} \psi_{\rho} \\
= & 0 .
\end{aligned}
$$

\footnotetext{
${ }^{4}$ The causality study for various couplings to external fields in [17] did not include non-minimal couplings; none of the models considered there was causal either.

${ }^{5}$ Our terminology is as follows: For a first order system devoid of gauge invariances, the primary (Lagrangian) constraints are simply any field equations without time derivatives. Requiring that the primary constraints are preserved by time evolution leads to secondary constraints and so forth. Precisely the same constraints arise as second class secondary and tertiary constraints, respectively, in a canonical Dirac analysis [18].
}

In particular, in temporal gauge $A_{0}=0$, the equations of motion for the spatial components of the Rarita-Schwinger field are

$$
\begin{aligned}
\gamma_{0} R_{i}= & \dot{\psi}_{i}+\gamma_{0}\left(\vec{\gamma} \cdot \overrightarrow{\mathcal{D}}-\frac{1}{2} m\right) \psi_{i}-\mathcal{D}_{i} \gamma_{0} \gamma \cdot \psi \\
& +\frac{i e}{2 m} \gamma_{0} \gamma_{\nu} \gamma_{i} \mathcal{F}^{\nu \rho} \psi_{\rho}=0 .
\end{aligned}
$$

We now obtain a secondary Lagrangian constraint from

$$
\begin{aligned}
\Theta_{2} \equiv & \mathcal{D} \cdot R=-\frac{3}{2} m^{2} \gamma \cdot \psi+\frac{i e}{2} \gamma_{\mu \nu \rho} F^{\mu \nu} \psi^{\rho}+\frac{i e}{m} \mathcal{D} \cdot \mathcal{F} \cdot \psi \\
= & -\frac{3}{2} m^{2} \gamma \cdot \psi+\frac{i e}{2} \gamma_{\mu \nu \rho} F^{\mu \nu} \psi^{\rho} \\
& +\frac{i e}{m}\left(\mathcal{D}^{i} \mathcal{F}_{i \nu}+\frac{1}{2} m \gamma^{0} \mathcal{F}_{0 \nu}\right) \psi^{\nu} \\
& +\frac{i e}{m}\left(\dot{\mathcal{F}}^{0 i} \psi_{i}+\mathcal{F}^{0 i} \dot{\psi}_{i}\right)=0 .
\end{aligned}
$$

Since $\dot{\psi}_{i}$ may be eliminated via Eq. $(33), \Theta_{2}$ constitutes a second independent algebraic relation amongst field components. [Again, observe that Eq. (34) would contain a term ( ie $/ m) \mathcal{F}_{00} \dot{\psi}_{0}$ for $\mathcal{F}_{00} \neq 0$ and become an equation of motion rather than a constraint.]

Upon substituting Eq. (33) into Eq. (34), we concentrate on the coefficient matrix $R$ of the Lagrange multiplier $\psi_{0}$ in $\Theta_{2}$, since $\psi_{0}$ must be determined by this relation:

$$
\begin{aligned}
\Theta_{2} \equiv & \gamma^{0} R \psi_{0}+\cdots \\
R= & -\frac{3}{2} m^{2}+\frac{i e}{2} \gamma_{i} F^{i j} \gamma_{j}-\frac{i e}{m} \gamma_{0}\left[\mathcal{D}_{i}, \mathcal{F}^{0 i}\right] \\
& -\frac{e^{2}}{2 m^{2}} \gamma_{0} \mathcal{F}^{0 i} \gamma_{0} \gamma_{j} \gamma_{i} \mathcal{F}{ }^{0 j} .
\end{aligned}
$$

In terms of the electric and magnetic fields $\left(E^{i}=F^{0 i}, B^{i}\right.$ $\left.=\widetilde{F}^{0 i}\right)$,

$$
\mathcal{F}^{0 i}=l_{1} E^{i}+\left(2 l_{2} \delta^{i j}+l_{5} \epsilon^{i j k} \gamma_{0} \gamma^{5} \gamma_{k}\right)\left(E_{j}-i \gamma^{5} B_{j}\right)
$$

Hence the critical $R$ whose loss of invertibility would leave $\psi_{0}$ (partly) undetermined, is 


$$
\begin{aligned}
R= & -\frac{3}{2} m^{2}-e\left[1-2 l_{2}\right] \gamma_{0} \gamma^{5} \vec{\gamma} \cdot \vec{B}-2 e l_{5} \gamma_{0} \vec{\gamma} \cdot \vec{E} \\
& +\frac{e^{2}}{2 m^{2}}\left(\left[l_{1}+2 l_{2}\right]^{2} \vec{E}^{2}+\left[2 l_{2}\right]^{2} \vec{B}^{2}\right) \\
& +\frac{e^{2}}{m^{2}}\left[2 l_{2}\left(l_{1}+2 l_{2}\right)+2 l_{5}^{2}\right] \gamma_{0} \vec{\gamma} \cdot(\vec{E} \times \vec{B}) \\
& +\frac{2 e^{2}}{m^{2}} l_{1} l_{5} \gamma^{5} \vec{E} \cdot \vec{B}-\frac{i e}{m}\left[l_{1}+2 l_{2}\right] \gamma_{0} \vec{\nabla} \cdot \vec{E} \\
& +\frac{i e}{m} l_{5} \gamma^{5} \vec{\gamma} \cdot\left(\vec{\nabla} \times \vec{E}-i \gamma^{5} \vec{\nabla} \times \vec{B}\right) .
\end{aligned}
$$

Pathologies in quantization and causality of the model thus occur whenever $\operatorname{det} R=0$ as a function of the background fields. To see how this occurs consider first a pure constant electric background. The determinant obviously develops a zero for a critical value of the electric field (with the choice $\left.l_{5}=0\right)$

$$
\vec{E}^{2}=3\left(\frac{m^{2}}{e\left[l_{1}+2 l_{2}\right]}\right)^{2}
$$

and the model is acausal. The choice of parameters $l_{1}+2 l_{2}$ $=0$ and $l_{5}$ arbitrary, cannot yield a causal model either: The determinant vanishes in a pure magnetic background for any values $l_{i}$ whenever

$$
-\frac{3}{2} m^{2}+\frac{e^{2}}{2 m^{2}}\left[2 l_{2}\right]^{2} \vec{B}^{2}= \pm e\left[1-2 l_{2}\right]|\vec{B}|
$$

This equation has a solution whenever the quadratic

$$
P(\beta) \equiv\left(2 l_{2} \beta\right)^{2} \pm 2\left(1-2 l_{2}\right) \beta-3=0, \quad \beta \equiv|e \vec{B}| / m^{2},
$$

has a solution for $\beta>0$. Clearly, for any non-zero value of $l_{2}, P(\beta)$ is positive for large enough $\beta$ and negative near $\beta=0$ so it always has a zero for some positive $\beta$ : All models, minimal or non-minimal, exhibit pathological behavior. ${ }^{6}$

Note that the supergravity-inspired and minimal coupling model have critical field values $\vec{B}^{2}=3 m^{4} / e^{2}$ and $\vec{B}^{2}$ $=\left(3 m^{2} / 2 e\right)^{2}$, respectively (the latter being the well known result of $[5,6])$. These bounds cannot be expressed in terms of the Lorentz invariants $\vec{E}^{2}-\vec{B}^{2}$ and $\vec{E} \cdot \vec{B}$, since the vector $\xi_{\mu}$ normal to the characteristic specifies a preferred time direction. The bounds themselves may, of course, be rephrased covariantly: For example Eq. (38) is a bound on $\left(F^{\mu \nu} \xi_{\nu}\right)^{2}$ which must hold for all timelike vectors $\xi_{\mu}$.

\footnotetext{
${ }^{6}$ Precisely the same analysis for a pure electric field including arbitrary $l_{5}$ yields the same result. Also even an uncharged (real) Majorana field, with only $l_{5} \neq 0$, displays acausal propagation.
}

It is interesting to speculate whether further non-minimal couplings may restore causality. In particular, the choice $l_{2}$ $=1 / 2, l_{5}=0$ at least removes the terms linear in $\vec{B}$ and $\vec{E}$ in Eq. (37) responsible for the original pathology of the minimal model. [In this respect, we note that this choice along with $l_{1}=-2$ is that of supergravity for which the fielddependent terms of Eq. (37) are proportional to the electromagnetic energy density, Poynting vector and charge density and for this reason causality is preserved there, upon taking gravity and the electromagnetic field dynamical.] However, in Appendix B we generalize Eq. (37) to arbitrary nonminimal couplings and show that broad classes of couplings fail to propagate causally.

Finally, as promised, we briefly review the argument linking the appearance of zeroes in $\operatorname{det} R$ to acausal propagation in the field equations. The computation of [6] amounts to studying the Cauchy problem of Eq. (29) and solving for the characteristic surfaces that determine the maximal speed of propagation. ${ }^{7}$ This is simply achieved by recalling that characteristics are determined by discontinuities of the highest order derivative terms appearing in the equations of motion [19]. If we denote the discontinuity of the first derivative of the Rarita-Schwinger field across the characteristic by

$$
\left[\partial_{\mu} \psi_{\nu}\right]=\xi_{\mu} \Psi_{\nu}
$$

where $\Psi_{\nu}$ is a non-zero vector-spinor field, then causal propagation forbids timelike $\xi_{\mu}$. However from the field equation (29) and its gamma-trace (31) we learn

$$
\begin{aligned}
{\left[R_{\mu}-\frac{1}{2} \gamma_{\mu} \gamma \cdot R\right] } & =\gamma^{\nu}\left(\xi_{\nu} \Psi_{\mu}-\xi_{\mu} \Psi_{\nu}\right) \\
{[\gamma \cdot R] } & =2(\gamma \cdot \xi \gamma \cdot \Psi-\xi \cdot \Psi)
\end{aligned}
$$

and in turn

$$
\xi^{2} \Psi_{\mu}=\xi_{\mu} \xi \cdot \Psi
$$

Proceeding by contradiction we take $\xi_{\mu}=(1,0,0,0)$ (timelike) without loss of generality since the original (29) is Lorentz covariant. We now need only study the leading discontinuities in time derivatives and in particular

$$
\left[\dot{\Theta}_{2}\right]=\gamma^{0} R \Psi_{0}=0
$$

which admits no non-vanishing solution for $\Psi_{0}$ unless $\operatorname{det} R=0$, the condition studied above.

\section{DISCUSSION}

We have seen that the most general charged massive spin $3 / 2$ theory with non-minimal couplings linear in the electro-

\footnotetext{
${ }^{7}$ In more physical terms, this is akin to solving the equations of motion in a high energy eikonal limit $\psi_{\mu}=\Psi_{\mu} \exp (i t x \cdot \xi)$ with $t \rightarrow \infty$. Clearly, solutions for $\xi_{\mu}$ timelike indicate superluminal propagation [4].
} 
magnetic field strength is described by the two parameter family

$$
\begin{aligned}
\mathcal{L}= & -\bar{\psi}^{\mu} \gamma_{\mu \nu \rho} \mathcal{D}^{\nu} \psi^{\rho}+\frac{i e}{m} \bar{\psi}_{\mu} F^{\mu \nu} \psi_{\nu} \\
& +\frac{3 i e}{4 m}(g-2) \bar{\psi}_{\mu} F^{\mu \nu} \psi_{\nu}-\frac{2 i e l_{2}}{m} \bar{\psi}_{\mu} \gamma^{5} \widetilde{F}^{\mu \nu} \psi_{\nu},
\end{aligned}
$$

two of the other a priori admissible parameters being excluded by DOF consistency; the third, corresponding to a diagonal Majorana coupling, did not affect our low energy or causality results. The physical interpretation of the first three terms in Eq. (46) was provided by studying LETs. The first is the usual minimally coupled Rarita-Schwinger theory with intrinsic gyromagnetic ratio $g=2 / 3$. Minimal coupling for half integer systems yields only the spin $1 / 2$ contribution to the Lorentz generators, while the second coupling is the spin 1 Pauli term required for $g=2$. Although $g=2$ is required for low energy unitarity of amplitudes describing pure electromagnetic interactions, more general phenomenological applications deal with the case $g \neq 2$, and one may safely include the anomalous magnetic moment coupling given in the third term. [Recall that at low energy, a coupling to $F^{\mu \nu}$ is equivalent to a coupling to the full Lorentz generators up to a factor $1 / 3$, by virtue of the identity (20).]

The fourth term is more subtle, as it does not contribute at low energy until quadrupole order. It will be an exercise of some physical importance to relate $l_{2}$, as well as effective Lagrangians including gradients of $F^{\mu \nu}$, to multipole moments along the lines of the method presented here for the magnetic dipole. The values $g=2$ and $l_{2}=1 / 2$, implying tree unitarity, represent a truncation of $N=2$ supergravity [along the lines of [4], but maintaining the correct DOF].

Our study of causality showed that no model maintaining the correct DOF avoids sharing the pathology of the minimal one. In fact this result applies to a very broad class of nonminimal couplings [beyond just linear in the field strength]; the criteria described in Sec. IV determine the causality of any non-minimally coupled model. An interesting issue under study [10] is whether including gravity dynamically can improve upon this situation; certainly for supergravities [8] this is the case, although the minimal model in curved space is known to still suffer the usual difficulties [20].

Finally, and perhaps most physically relevant, the models we have studied, despite the formal causal pathologies of the interacting fields, provide a useful parametrization for an effective low energy description of higher spin excitations: They are a field theoretical framework for the generic LET properties.

\section{ACKNOWLEDGMENTS}

We are indebted to H. Schnitzer and S. Weinberg for reminding us of the Wigner-Eckart theorem's universality and thank also M. Porrati and P. van Nieuwenhuizen for discussions. This work was supported by the National Sci- ence Foundation under grant PHY99-73935 and by the Australian Research Council.

\section{APPENDIX A: HIGHER SPIN SOFT PHOTON VERTICES}

The results of Sec. III A are easily generalized to arbitrary higher spin targets. For higher integer spin $s$ we employ a complex symmetric tensor field $\phi_{\mu_{1} \cdots \mu_{s}}$. On-shell, $\phi_{\mu_{1} \cdots \mu_{s}}$ is asymptotic to a free field satisfying

$$
\left(\square-m^{2}\right) \phi_{\mu_{1} \cdots \mu_{s}}=0=\phi_{\mu}{ }^{\mu} \mu_{3} \cdots \mu_{s}=\partial_{\mu} \phi_{\mu_{2} \cdots \mu_{s}} \text {. }
$$

The corresponding half integer spin $s \equiv n+\frac{1}{2}$ representation is a complex Dirac symmetric tensor-spinor $\psi_{\mu_{1} \cdots \mu_{n}}$ obeying free field equations

$$
\begin{aligned}
(\not+m) \psi_{\mu_{1} \cdots \mu_{n}} & =0=\gamma_{\mu} \psi^{\mu}{ }_{\mu_{2} \cdots \mu_{n}}=\psi_{\mu}{ }^{\mu}{ }_{\mu_{3} \cdots \mu_{n}} \\
& =\partial_{\mu} \psi_{\mu_{2} \cdots \mu_{n}}^{\mu} .
\end{aligned}
$$

Here, as for spin $3 / 2$, it is essential to determine appropriate non-minimal couplings to lower trace and gamma-trace field components in seeking a correct DOF count in an electromagnetic background [10]. In this appendix however, we ignore these couplings since they are irrelevant to the soft photon vertex.

The general Lagrangian is the sum of the minimally coupled massive higher spin $\operatorname{action}^{8}$ of [21] plus the most general non-minimal couplings (ignoring trace couplings) denoted by $\mathcal{L}_{\mathrm{NM}}$. For integer spins, ${ }^{9}$

$$
\mathcal{L}_{\mathrm{NM}}=-i e l_{1} \phi_{\mu}^{*} F^{\mu \nu} \phi_{\nu}
$$

and for half integer spins [the precise analogue of Eq. (2)],

$$
\mathcal{L}_{\mathrm{NM}}=-\frac{i e}{m}\left[l_{1} \bar{\psi}_{\mu} F^{\mu \nu} \psi_{\nu}+l_{2} \bar{\psi} \hat{F} \psi\right]
$$

in the terse notation, appropriate for bilinears, that drops any indices contracted directly between a field and its complex conjugate (so that, for example, $\bar{\psi} \hat{F} \psi$ $\left.\equiv \bar{\psi}_{\rho_{1} \cdots \rho_{n}} \hat{F} \psi^{\rho_{1} \cdots \rho_{n}}\right)$.

The LET for the emission of a single photon for arbitrary spin target is

$$
T_{f i}=\frac{i \mu}{2 s} F_{\mu \nu} \mathcal{M}^{\mu \nu}+\mathcal{O}\left(\omega^{2}\right)
$$

\footnotetext{
${ }^{8}$ Note that in [21] the action is in terms of traceless and gammatraceless fields symmetric in vector indices along with auxiliary fields. Using field redefinitions one may work, equivalently, with unconstrained symmetric fields and a reduced set of auxiliaries as above.

${ }^{9}$ Here and throughout, we have ignored derivative couplings, although they may also contribute to the magnetic moment; this is illustrated in [22].
} 
where now the Lorentz generators in a higher spin representation are given by

$$
\mathcal{M}_{\mu \nu}=2 m s \varepsilon_{[\mu}^{\prime *} \varepsilon_{\nu]}
$$

for integer spins, and

$$
\mathcal{M}_{\mu \nu}=\frac{1}{2} \bar{u}^{\prime} \gamma_{\mu \nu} u+2 s \bar{u}_{[\mu}^{\prime} u_{\nu]}
$$

for half integer spins. The initial, $\varepsilon_{\mu_{1} \ldots \mu_{s}}$ and $u_{\mu_{1} \ldots \mu_{n}}$, respectively, and final target polarizations (with primes) satisfy the usual conditions implied by Eqs. (A1) and (A2).

We must now compute the on-shell vertices in the soft photon limit and compare the results with the LET (A5). The integer spin case is simple and we find a gyromagnetic ratio

$$
g=\frac{1}{s}-\frac{2 l_{1}}{s}
$$

where we have included a contribution $g=1 / s$ from the minimally coupled Lagrangian. (Of course, unlike the half integer case, a second order system is well known to be ambiguous due to possible partial integrations before minimal coupling. In fact the minimal model alone can yield any gyromagnetic ratio between $g=0$ and $g=1 / s$, the case quoted above being attained by writing the Lagrangian in first order form and only thereafter coupling minimally [21].)

The half integer case directly follows Sec. III A; we must include the minimal interaction

$$
\mathcal{L}_{\min }^{\mathrm{int}}=-i e \bar{\psi} A \psi
$$

to which the Gordon identity (18) may be applied unaltered. Once again, there is a special model which requires no further low energy identities to fulfill the LET, namely the one with $n l_{1}=4 l_{2}-1$. The LET is satisfied, of course, for all parameters $\left(l_{1}, l_{2}\right)$ as follows from the obvious generalization of the identity (20), implying

$$
\mathcal{M}_{\mu \nu}=s \bar{u}^{\prime} \gamma_{\mu \nu} u+\mathcal{O}(\omega) \text {. }
$$

As a result one finds

$$
g=\frac{1}{s}-\frac{2\left(l_{1}+2 l_{2}\right)}{s},
$$

which clearly reproduces Eq. (21) for $s=3 / 2$. Note that the higher spin analogue of Eq. (7) again implies that an $i \gamma^{5} \widetilde{F}_{\mu \nu}$ coupling does not contribute at linear order in $\omega$, so that low energy physics is encapsulated by a single magnetic moment coupling.

\section{APPENDIX B: CAUSALITY OF GENERAL NON-MINIMAL MODELS}

The curious reader may wonder whether there exist causal non-minimal couplings for more general functions $\mathcal{F}^{\mu \nu}$ of field strengths. The most general antisymmetric (so that DOFs are maintained) one is $\mathcal{F}^{\mu \nu}=W^{\mu \nu}+i \gamma^{5} X^{\mu \nu}+i \gamma^{\mu \nu} Y$ $+\gamma^{5} \gamma^{\mu \nu} Z$ where $W^{\mu \nu}$ and $Y$ are parity even, $X^{\mu \nu}$ and $Z$ are parity odd, all built from $F_{\mu \nu}, \widetilde{F}_{\mu \nu},\left(F_{\rho \sigma} F^{\rho \sigma}\right),\left(F_{\rho \sigma} \widetilde{F}^{\rho \sigma}\right)$ and field-gradient dependent terms. [For brevity we omit the diagonal, Majorana, $l_{5}$ term.] Causality is determined by substituting this expansion into the matrix (35) and searching for zeroes in its determinant. If we set $Z=0$ [in any case $Z$ must be an odd function of the axial scalar $\left(F_{\rho \sigma} \widetilde{F}^{\rho \sigma}\right)$, so vanishes for pure electric or pure magnetic field configurations] then the analysis of this determinant is almost identical to that performed above: The third term $Y$ is a fielddependent mass term so replacing the operator $\mathcal{D}_{\mu} \rightarrow D_{\mu}$ $+\frac{1}{2} m_{*} \gamma_{\mu}$ where $m_{*}=m+Y$, calling $\mathcal{F}^{i 0}=E_{*}^{i}+i \gamma^{5} B_{*}^{i}$ $\left(E_{*}^{i}=W^{i 0}, B_{*}^{i}=X^{i 0}\right)$ and derivatives of $m_{*}$ no longer vanish. The matrix $R$ then reads

$$
\begin{aligned}
R= & -\frac{3}{2} m_{*}^{2}+\frac{e^{2}}{2 m^{2}}\left(\vec{E}_{*}^{2}+\vec{B}_{*}^{2}\right)+e \gamma_{0} \gamma^{5} \vec{\gamma} \cdot\left(\vec{B}+\frac{m_{*}}{m} \vec{B}_{*}\right) \\
& +\frac{i e}{m} \gamma_{0}\left(\vec{\nabla} \cdot \vec{E}_{*}-i \gamma^{5} \vec{\nabla} \cdot \vec{B}_{*}\right) \\
& +\vec{\gamma} \cdot \vec{\nabla} m_{*}-\frac{e^{2}}{m^{2}} \gamma_{0} \vec{\gamma} \cdot\left(\vec{E}_{*} \times \vec{B}_{*}\right) .
\end{aligned}
$$

For the (simplest) case $F_{\mu \nu}$ constant, $\operatorname{det} R$ vanishes whenever

$$
\begin{aligned}
& {\left[-\frac{3}{2} m_{*}^{2}+\frac{e^{2}}{2 m^{2}}\left(\vec{E}_{*}^{2}+\vec{B}_{*}^{2}\right)\right]^{2}} \\
& -\left[e\left(\vec{B}+\frac{m_{*}}{m} \vec{B}_{*}\right) \pm \frac{e^{2}}{m^{2}} \vec{E}_{*} \times \vec{B}_{*}\right]^{2}=0 .
\end{aligned}
$$

Observe that for $Y=0=Z$, i.e., $m_{*}=m$, and with $\mathcal{F}^{\mu \nu}$ growing unboundedly for large $\mathcal{F}^{\mu \nu}$, the model is not causal: For $\mathcal{F}^{\mu \nu}=0$ the expression (B2) is positive but the first term in square brackets must have a zero for large enough $\vec{E}_{*}$ or $\vec{B}_{*}$ at which point the second term is necessarily negative, and solutions to Eq. (B2) will exist. While this shows that broad classes of generalized couplings remain acausal, the above completely general criterion can applied to a systematic search for causal models.
[1] S. Weinberg, in Lectures on Elementary Particles and Quantum Field Theory, Vol. 1, Brandeis University Summer Institute 1970, edited by S. Deser, M. Grisaru, and H. Pendleton (M.I.T. Press, Cambridge, MA, 1970).

[2] F. Belinfante, Phys. Rev. 92, 997 (1953); K. Case, ibid. 94,
1442(Z6) (1954).

[3] J. M. Cornwall, D. N. Levin, and G. Tiktopoulos, Phys. Rev. D 10, 1145 (1974); C. H. Llewellyn Smith, Phys. Lett. 46B, 233 (1973).

[4] S. Ferrara, M. Porrati, and V. L. Telegdi, Phys. Rev. D 46, 
3529 (1992).

[5] K. Johnson and E. C. Sudarshan, Ann. Phys. (N.Y.) 13, 126 (1961).

[6] G. Velo and D. Zwanziger, Phys. Rev. 186, 1337 (1969).

[7] D. Z. Freedman and A. Das, Nucl. Phys. B120, 221 (1977).

[8] S. Deser and B. Zumino, Phys. Rev. Lett. 38, 1433 (1977).

[9] A. Shamaly and A. Z. Capri, Ann. Phys. (N.Y.) 74, 503 (1972).

[10] S. Deser and A. Waldron (in progress).

[11] S. Ferrara and P. van Nieuwenhuizen, Phys. Rev. Lett. 37, 1669 (1976).

[12] F. E. Low, Phys. Rev. 96, 1428 (1954); M. Gell-Mann and M. L. Goldberger, ibid. 96, 1433 (1954).

[13] L. Lapidus and C. Kuang-Chao, Zh. Éksp. Teor. Fiz. 39, 1286 (1961) [Sov. Phys. JETP 12, 898 (1961)]; K. Bardakci and H. Pagels, Phys. Rev. 166, 1783 (1968); S. Saito, ibid. 184, 1894 (1969).

[14] P. Auvil and J. Brehm, Phys. Rev. 145, 1156 (1966); T. Moroi, hep-ph/9503210.

[15] Elementary diagrams are rapidly computed with the algebraic manipulation program FORM: J. A. M. Vermaseren, Computer Algebra Nederland, Amsterdam, 1991.

[16] F. Piccinini, G. Venturi, and R. Zucchini, Lett. Nuovo Cimento Soc. Ital. Fis. 41, 536 (1984).

[17] C. R. Hagen and L. P. Singh, Phys. Rev. D 26, 393 (1982).

[18] Details of the canonical formulation for massive spin 3/2 may be found in A. Hasumi, R. Endo, and T. Kimura, J. Phys. A 12, L217 (1979); K. Inoue, M. Omote, and M. Kobayashi, Prog. Theor. Phys. 63, 1413 (1980); M. Yamada and W. Cox, J. Phys. A 22, 1599 (1989); V. Pascalutsa, Phys. Rev. D 58, 096002 (1998).

[19] J. Madore and W. Tait, Commun. Math. Phys. 30, 201 (1973).

[20] J. Madore, Phys. Lett. 55B, 217 (1975).

[21] L. P. Singh and C. R. Hagen, Phys. Rev. D 9, 898 (1974).

[22] J. Schwinger, Particles, Sources, and Fields (Addison-Wesley, Reading, MA, 1970), Vol. I. 